\title{
Molecular Profiling
}

National Cancer Institute

\section{Source}

National Cancer Institute. Molecular Profiling. NCI Thesaurus. Code C19771.

Analysis of the RNA expression pattern in a tissue sample using molecular techniques. 